\title{
Generalized Gray Codes for Local Rank Modulation
}

\author{
Eyal En Gad \\ Elec. Eng. \\ Caltech \\ Pasadena, CA 91125, U.S.A. \\ eengad@caltech.edu
}

\author{
Michael Langberg \\ Comp. Sci. Division \\ Open University of Israel \\ Raanana 43107, Israel \\ mikel@openu.ac.il
}

\author{
Moshe Schwartz \\ Elec. and Comp. Eng. \\ Ben-Gurion University \\ Beer Sheva 84105, Israel \\ schwartz@ee.bgu.ac.il
}

\author{
Jehoshua Bruck \\ Elec. Eng. \\ Caltech \\ Pasadena, CA 91125, U.S.A. \\ bruck@paradise.caltech.edu
}

\begin{abstract}
We consider the local rank-modulation scheme in which a sliding window going over a sequence of real-valued variables induces a sequence of permutations. Local rankmodulation is a generalization of the rank-modulation scheme, which has been recently suggested as a way of storing information in flash memory.

We study Gray codes for the local rank-modulation scheme in order to simulate conventional multi-level flash cells while retaining the benefits of rank modulation. Unlike the limited scope of previous works, we consider code constructions for the entire range of parameters including the code length, sliding window size, and overlap between adjacent windows. We show our constructed codes have asymptotically-optimal rate. We also provide efficient encoding, decoding, and next-state algorithms.
\end{abstract}

\section{INTRODUCTION}

With the recent application to flash memories, the rankmodulation scheme has gained renewed interest as evident in the recent series of papers [9], [15], [16], [18], [20], [22]. In the conventional modulation scheme used in flash-memory cells, the absolute charge level of each cell is quantized to one of $q$ levels, resulting in a single demodulated symbol from an alphabet of size $q$. In contrast, in the rank modulation scheme a group of $n$ flash cells comprise a single virtual cell storing a symbol from an alphabet of size $n$ !, where each symbol is assigned a distinct configuration of relative charge levels in the $n$ cells. Thus, there is no more need for threshold values to distinguish between various stored symbols, which mitigates the effects of retention in flash cells (slow charge leakage). In addition, if we allow only a simple programming (charge-injection) mechanism called "push-tothe-top", whereby a single cell is driven above all others in terms of charge level, then no over-programming can occur, a problem which considerably slows down programming in conventional multi-level flash cells.

Rank modulation has been studied intermittently since the early works of Slepian [19] (later extended in [1]), in which permutations were used to digitize vectors from a time-discrete memoryless Gaussian source, and Chadwick and Kurz [6], in which permutations were used in the context of signal detection over channels with non-Gaussian noise (especially

This work was supported in part by ISF grant 134/10, ISF grant 480/08, the Open University of Israel's research fund (grant no. 46114), the NSF grant ECCS-0802107, and an NSF-NRI award. impulse noise). Other works on the subject include [1][3], [5], [7], [8]. More recently, permutations were used for communicating over powerlines (for example, see [21]), and for modulation schemes for flash memory [15], [16], [20], [22].

One drawback to the rank-modulation scheme is the fact that we need to reconstruct the permutation induced by the relative charge levels of the participating cells. If $n$ cells are involved, at least $\Omega(n \log n)$ comparisons are needed, which might be too high for some applications. It was therefore suggested in [9], [18], [22] that only local comparisons be made, creating a sequence of small induced permutations instead of a single all-encompassing permutation. This obviously restricts the number of distinct configurations, and thus, reduces the size of the resulting alphabet as well. In the simplest case, requiring the least amount of comparisons, the cells are located in a one-dimensional array and each cell is compared with its two immediate neighbors requiring a single comparator between every two adjacent cells [9], [18].

Yet another drawback of the rank-modulation scheme is the fact that distinct $n$ charge levels are required for a group of $n$ physical flash cells. Therefore, restricted reading resolution prohibits the use of large values of $n$. However, when only local views are considered, distinct values are required only within a small local set of cells, thus enabling the use of large groups of cells with local rank modulation.

An important application for rank-modulation in the context of flash memory was described in [15]: A set of $n$ cells, over which the rank-modulation scheme is applied, is used to simulate a single conventional multi-level flash cell with $n$ ! levels corresponding to the alphabet $\{0,1, \ldots, n !-1\}$. The simulated cell supports an operation which raises its value by 1 modulo $n$ !. This is the only required operation in many rewriting schemes for flash memories (see [4], [12]-[14], [23]), and it is realized in [15] by a Gray code traversing the $n$ ! states where, physically, the transition between two adjacent states in the Gray code is achieved by using a single "pushto-the-top" operation. In the context of local rank modulation, Gray codes for the local scheme were studied in [9], [18], where necessary conditions as well as constructions were provided.

Having considered the two extremes: full rank modulation with a single permutation of $n$ cells, and extreme local 


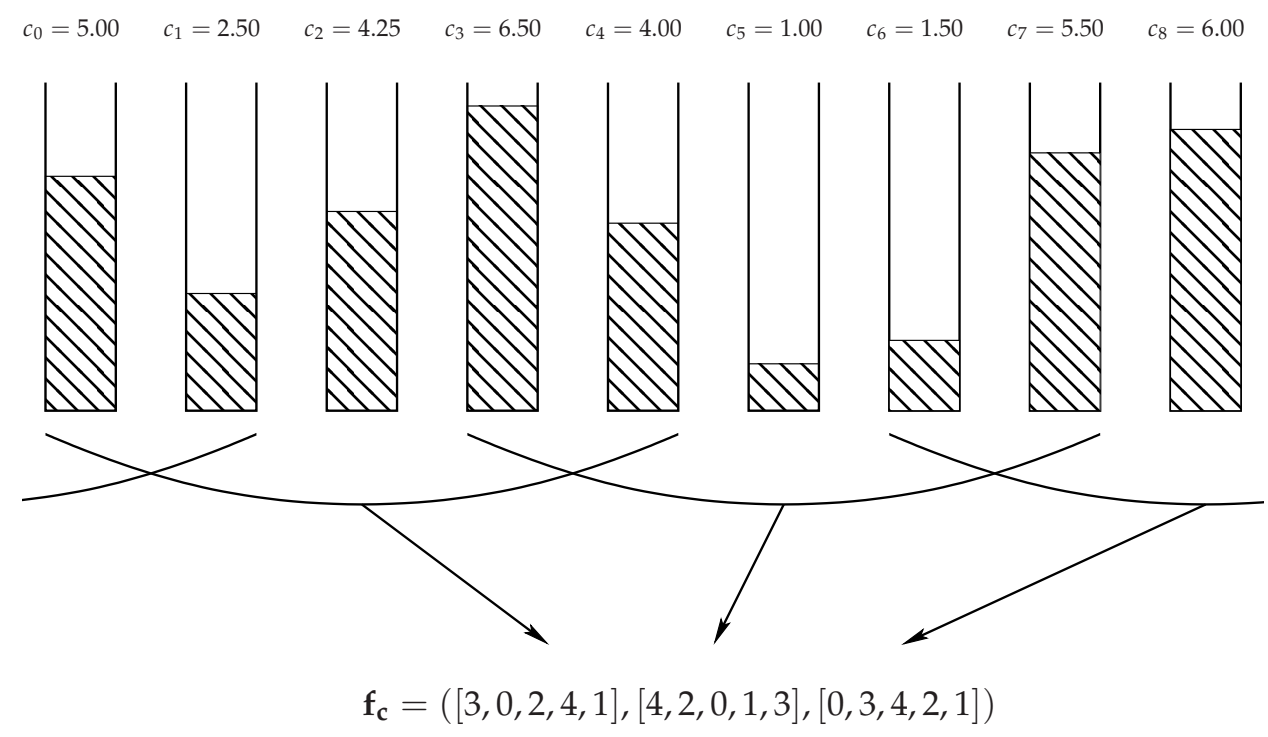

Figure 1. Demodulating a $(3,5,9)$-locally rank-modulated signal.

rank modulation with a sequence of $n$ permutations over 2 elements, the question of whether any middle-road solutions exist remains open. We address this question in this paper by considering the generalized local rank modulation scheme in which a sequence of several permutations of a given size provide the local views into ranking of the cells. We construct Gray codes for this scheme which asymptotically achieve the maximum possible rate, and consider efficient encoding/decoding algorithms, as well as efficient next-state computation.

The rest of the paper is organized as follows. In Section I we give preliminary definitions and notation. In Section present our construction for optimal local rank modulation for general degrees of locality. We conclude with a discussion in Section IV

\section{DEFINITIONS AND Notation}

We shall now proceed to introduce the notation and definitions pertaining to local rank modulation and Gray codes. We will generally follow the notation introduced in [9], [18].

\section{A. Local Rank Modulation}

Let us consider a sequence of $t$ real-valued variables, $\mathbf{c}=\left(c_{0}, c_{1}, \ldots, c_{t-1}\right) \in \mathbb{R}^{t}$, where we further assume $c_{i} \neq c_{j}$ for all $i \neq j$. The $t$ variables induce a permutation $f_{\mathrm{c}} \in S_{t}$, where $S_{t}$ denotes the set of all permutations over $[t]=$ $\{0,1,2, \ldots, t-1\}$. The permutation $f_{\mathbf{c}}$ is defined as

$$
f_{\mathbf{c}}(i)=\left|\left\{j \mid c_{j}<c_{i}\right\}\right| \text {. }
$$

Loosely speaking, $f_{\mathbf{c}}(i)$ is the rank of the $i$ th cell in ascending order. This ranking is equivalent to the permutation described in [9], [18], though different.

Given a sequence of $n$ variables, $\mathbf{c}=\left(c_{0}, c_{1}, \ldots, c_{n-1}\right)$, we define a window of size $t$ at position $p$ to be

$$
\mathbf{c}_{p, t}=\left(c_{p}, c_{p+1}, \ldots, c_{p+t-1}\right)
$$

where the indices are taken modulo $n$, and also $0 \leqslant p \leqslant n-1$, and $1 \leqslant t \leqslant n$. We now define the $(s, t, n)$-local rankmodulation (LRM) scheme, which we do by defining the demodulation process. Let $s \leqslant t \leqslant n$ be positive integers, with $s \mid n$. Given a sequence of $n$ distinct real-valued variables, $\mathbf{c}=\left(c_{0}, c_{1}, \ldots, c_{n-1}\right)$, the demodulation maps $\mathbf{c}$ to the sequence of $n / s$ permutations from $S_{t}$ as follows:

$$
\mathbf{f}_{\mathbf{c}}=\left(f_{\mathbf{c}_{0, t}}, f_{\mathbf{c}_{s, t}}, f_{\mathbf{c}_{2 s, t}}, \ldots, f_{\mathbf{c}_{n-s, t}}\right) \text {. }
$$

Loosely speaking, we scan the $n$ variables using windows of size $t$ positioned at multiples of $s$ and write down the permutations from $S_{t}$ induced by the local views of the sequence.

In the context of flash-memory storage devices, we shall consider the $n$ variables, $\mathbf{c}=\left(c_{0}, c_{1}, \ldots, c_{n-1}\right)$, to be the charge-level readings from $n$ flash cells. The demodulated sequence, $\mathbf{f}_{\mathrm{c}}$, will stand for the original information which was stored in the $n$ cells. This approach will serve as the main motivation for this paper, as it was also for [9], [15], [16], [18], [20], [22]. See Figure 11 for an example.

We say a sequence $\mathbf{f}$ of $n / s$ permutations over $S_{t}$ is $(s, t, n)$ LRM realizable if there exists $\mathbf{c} \in \mathbb{R}^{n}$ such that $\mathbf{f}=\mathbf{f}_{\mathrm{c}}$, i.e., it is the demodulated sequence of $\mathbf{c}$ under the $(s, t, n)$-LRM scheme. Except for the degenerate case of $s=t$, not every sequence is realizable. We denote the set of all $(s, t, n)$-LRM realizable permutation sequences as $\mathcal{R}(s, t, n)$. In a later part of this section, we show that the number of states representable by an $(s, t, n)$-LRM scheme, i.e., the size of $\mathcal{R}(s, t, n)$, is roughly $(t \cdot(t-1) \cdot \ldots \cdot(t-s+1))^{n / s}$ (this fact is also stated in [22]).

While any $\mathbf{f} \in \mathcal{R}(s, t, n)$ may be represented as a sequence of $n / s$ permutations over $S_{t}$, a more succinct representation is possible based on the (mixed-radix) factoradic notation system (see [17] for the earliest-known definition, and [15] for a related use): We can represent any permutation 
$f=[f(0), \ldots, f(t-1)] \in S_{t}$ with a sequence of digits $d_{t-1}, d_{t-2}, \ldots, d_{1}, d_{0}$, where $d_{i} \in \mathbb{Z}_{i}$, and $d_{i}$ counts the number of elements to the right of $f(i)$ which are of lower value. We call $d_{t-1}$ the most significant digit and $d_{0}$ the least significant digit. If $f=f_{\mathbf{c}}$ for some $\mathbf{c} \in \mathbb{R}^{t}$, then the factoradic representation is easily seen to be equivalent to counting the number of cells to the right of the $i$ th cell which are with lower charge levels.

Continuing with our succinct representation, we now contend that due to the overlap between local views, we can then represent each of the local permutations $f_{\mathbf{c}_{i \cdot s, t}}$ using only the $s$ most-significant digits in their factoradic notation. We denote this (partial) representation as $\bar{f}_{\mathbf{c}_{i \cdot s, t}}$. Accordingly, we define,

$$
\overline{\mathbf{f}}_{\mathbf{c}}=\left(\bar{f}_{\mathbf{c}_{0, t}}, \bar{f}_{\mathbf{c}_{s, t}}, \bar{f}_{\mathbf{c}_{2 s, t}}, \ldots, \bar{f}_{\mathbf{c}_{n-s, t}}\right),
$$

and the set of all such presentations as $\overline{\mathcal{R}}(s, t, n)$. Thus, for example, the configuration of Figure 1 would be represented by $((3,0,1),(4,2,0),(0,2,2))$.

Lemma 1. For all $1 \leqslant s \leqslant t \leqslant n$,

$$
|\overline{\mathcal{R}}(s, t, n)| \leqslant|\mathcal{R}(s, t, n)| \leqslant(t-s) ! \cdot\left(\frac{t !}{(t-s) !}\right)^{\frac{n}{s}}
$$

Proof: That $|\overline{\mathcal{R}}(s, t, n)| \leqslant|\mathcal{R}(s, t, n)|$ is trivial, since any $\mathbf{f} \in \mathcal{R}(s, t, n)$ results in one $\overline{\mathbf{f}} \in \overline{\mathcal{R}}(s, t, n)$. For the other inequality, assume we fix the permutation induced by the first $t-s$ cells, where there are $(t-s)$ ! ways of doing so. It follows that there are $t ! /(t-s)$ ! ways of choosing $f_{\mathbf{c}_{n-s, t}}$, and then the same amount of ways of choosing $f_{\mathbf{c}_{n-2 s, t}}$, and continuing all the way up to $f_{\mathbf{c}_{0, t}}$ we get the desired result.

When $s=t=n$, the $(n, n, n)$-LRM scheme degenerates into a single permutation from $S_{n}$. This was the case in most of the previous works using permutations for modulation purposes. A slightly more general case, $s=t<n$ was discussed by Ferreira et al. [10] in the context of permutation trellis codes, where a binary codeword was translated tuplewise into a sequence of permutation with no overlap between the tuples. An even more general case was defined by Wang et al. [22] (though in a slightly different manner where indices are not taken modulo $n$, i.e., with no wrap-around). In [22], the sequence of permutations was studied under a chargedifference constraint called bounded rank-modulation, and mostly with parameters $s=t-1$, i.e., an overlap of one position between adjacent windows. Finally, using the same terminology as this paper, the case of $(1,2, n)$-LRM was considered in [9], [18].

\section{B. Gray Codes}

Generally speaking, a Gray code, $G$, is a sequence of distinct states (codewords), $G=g_{0}, g_{1}, \ldots, g_{N-1}$, from an ambient state space, $g_{i} \in S$, such that adjacent states in the sequence differ by a "small" change. What constitutes a "small" change usually depends on the code's application.

Since we are interested in building Gray codes for flash memory devices with the $(s, t, n)$-LRM scheme, our ambient space is $\mathcal{R}(s, t, n)$, which is the set of all realizable sequences under $(s, t, n)$-LRM.

The transition between adjacent states in the Gray code is directly motivated by the flash memory application, and was first described and used in [15], and later also used in [9], [18]. This transition is the "push-to-the-top" operation, which takes a single flash cell and raises its charge level above all others.

In our case, however, since we are considering a local rankmodulation scheme, the "push-to-the-top" operation merely raises the charge level of the selected cell above those cells which are comparable with it. Thus, we define the set of allowed transitions as $T=\left\{\tau_{0}, \tau_{1}, \ldots, \tau_{n-1}\right\}$, which is a set of functions, $\tau_{j}: \mathcal{R}(s, t, n) \rightarrow \mathcal{R}(s, t, n)$, where $\tau_{j}$ represents a "push-to-the-top" operation performed on the $j$-th cell. More precisely, let $\mathbf{f}$ be an $(s, t, n)$-LRM realizable sequence of permutations, i.e., there exists $\mathbf{c} \in \mathbb{R}^{n}$ such that $\mathbf{f}=\mathbf{f}_{\mathbf{c}}$. Now define the transition $\tau_{j}$ acting on $\mathbf{f}$ as $\mathbf{f}^{\prime}=\mathbf{f}_{\mathrm{c}^{\prime}}^{\prime}$ realizable by the variables $\mathbf{c}^{\prime}=\left(c_{0}^{\prime}, \ldots, c_{n-1}^{\prime}\right) \in \mathbb{R}^{n}$ such that $c_{j}^{\prime}$ is pushed to a value higher than all of it's comparable cells. We denote $r(j)$ as the rightmost index (cyclically) among the cells that share a window with $c_{j}^{\prime}$, and $l(j)$ as the leftmost index (cyclically) among them. We can find $r(j)$ and $l(j)$ by the following expressions:

$$
\begin{aligned}
& l(j)=s\left\lceil\frac{j-t+1}{s}\right\rceil \bmod n, \\
& r(j)=\left(s\left\lfloor\frac{j}{s}\right\rfloor+(t-1)\right) \bmod n .
\end{aligned}
$$

Now $\mathbf{c}^{\prime}$ is given by the following expression:

$$
c_{i}^{\prime}= \begin{cases}c_{i} & i \neq j, \\ \max \left\{c_{l(i)}, \ldots, c_{r(i)}\right\}+1 & i=j .\end{cases}
$$

Definition 2. A Gray code $G$ for $(s, t, n)$-LRM (denoted $(s, t, n)$-LRMGC) is a sequence of distinct codewords, $G=$ $g_{0}, g_{1}, \ldots, g_{N-1}$, where $g_{i} \in \mathcal{R}(s, t, n)$. For all $0 \leqslant i \leqslant N-2$, we further require that $g_{i+1}=\tau_{j}\left(g_{i}\right)$ for some $j$. If $g_{0}=$ $\tau_{j}\left(g_{N-1}\right)$ for some $j$, then we say the code is cyclic. We call $N$ the size of the code, and say $G$ is optimal if $N=|\mathcal{R}(s, t, n)|$.

Definition 3. We say a family of codes, $\left\{G_{i}\right\}_{i=1}^{\infty}$, where $G_{i}$ is an $\left(s, t, n_{i}\right)$-LRMGC of size $N_{i}, n_{i+1}>n_{i}$, is asymptotically optimal if

$$
\lim _{i \rightarrow \infty} \frac{\log _{2} N_{i}}{\log _{2}\left|\mathcal{R}\left(s, t, n_{i}\right)\right|}=1 \text {. }
$$

\section{GRAY CODES FOR $(s, t, n)$-LRM}

In this section we present efficiently encodable and decodable asymptotically-optimal Gray codes for $(s, t, n)$-LRM. A rough description of our construction follows. First we partition the $n$ cells into $m$ blocks each of size $m / n$. To simplify our presentation we set $m=\sqrt{n}$, implying that we have $m$ blocks, each of size $m$. Denote the cells in block $i$ by $\mathbf{c}_{i}$. For each block $\mathbf{c}_{i}$ we will use the factoradic representation $\overline{\mathbf{f}}_{\mathbf{c}_{i}}$ to represent permutations in $\overline{\mathcal{R}}(s, t, m)$. Namely, each and 
every block can be thought of an element of an alphabet $\Sigma=\left\{v_{0}, \ldots, v_{V-1}\right\}$ of size $V$.

Now, consider any de-Bruijn sequence $S$ of order $m-1$ over $\Sigma$ (of period $V^{m-1}$ ). Namely, $S$ will consist of a sequence of $V^{m-1}$ elements $v_{S_{0}}, v_{S_{1}}, \ldots, v_{S_{V^{m-1}-1}}$ over $\Sigma$ such that the subsequences $v_{s_{i}}, \ldots, v_{s_{i+m-2}}$ of $S$ cover all $(m-1)$-tuples of $\Sigma$ exactly once, sub-indices of $s$ taken modulo $V^{m-1}$. Here, $s_{i} \in[V]$. Such sequences $S$ exist, e.g., [11].

We are now ready to construct our Gray code $G$. The construction will have two phases. First we construct so-called anchor elements in $G$, denoted as $\bar{G}=\left\{g_{0}, \ldots, g_{L-1}\right\}$. The elements of $\bar{G}$ will consist of a cyclic Gray code over $\Sigma^{m}$. That is, the difference between each $g_{i}$ and $g_{i+1}$ in $\bar{G}$ will be in only one out of the $m$ characters (from $\Sigma$ ) in $g_{i}$. Specifically, the code $\bar{G}$ will be derived from the de-Bruijn sequence $S$ as follows: we set $g_{0}$ to be the first $m$ elements of $S$, and in the transition from $g_{i}$ to $g_{i+1}$ we change $v_{S_{i}}$ to $v_{S_{i+m}}$. The code $\bar{G}$ is detailed below:

$$
\begin{aligned}
& \begin{array}{llllll}
g_{0} & =v_{s_{m-1}} & v_{s_{m-2}} & \cdots & v_{s_{1}} & \frac{v_{s_{0}}}{g_{1}}= \\
v_{s_{m-1}} & v_{s_{m-2}} & \cdots & \underline{v_{s_{1}}} & v_{s_{m}}
\end{array} \\
& g_{2}=\begin{array}{lllll}
v_{s_{m-1}} & v_{s_{m-2}} & \ldots & v_{s_{m+1}} & v_{s_{m}}
\end{array} \\
& g_{L-2}=v_{S_{L-1}} \quad \frac{v_{S_{L-2}}}{v_{S_{L-2}}} \ldots v_{S_{1}} \quad v_{S_{0}} \\
& g_{L-1}=\underline{v_{S_{L-1}}} \quad \begin{array}{lllll}
v_{S_{m-2}} & \ldots & v_{S_{1}} & v_{S_{0}}
\end{array}
\end{aligned}
$$

where $L=\operatorname{lcm}\left(m, V^{m-1}\right)$, the sub-indices of $s$ are taken modulo $V^{m-1}$, and the underline is an imaginary marking distinguishing the block which is about to change.

With the imaginary marking of the underline, the code $\bar{G}$ is clearly a Gray code over $\Sigma^{m}$ due to the properties of the de-Bruijn sequence $S$. However $\bar{G}$ does not suffice for our construction as the transitions between the anchors $g_{i}$ and $g_{i+1}$ involve changing the entries of an entire block, which may involve many push-to-the-top operations. We thus refine $\bar{G}$ by adding additional elements between each pair of adjacent anchors from $\bar{G}$ that allow us to move from the block configuration in $g_{i}$ to that in $g_{i+1}$ by a series of push-to-the-top operations. Our construction is summarized below formally.

Construction 1. We consider the $(s, t, n)-L R M, n$ be a square, $m=\sqrt{n} \geqslant t+2$, and require that $s \mid m$. Let $\left\{v_{0}, v_{1}, \ldots, v_{V-1}\right\}$ be a set of $V$ distinct mixed-radix vectors of length $m$ taken from $([t] \times[t-1] \times \cdots \times[t-s])^{m / s}$. The values of the last $s(\lceil(t+2) / s\rceil-1)$ digits of each $v_{i}$ do not play a role in the representation of the stored data and are called non-information digits, so by abuse of notation, a mixedradix vector $\left(r_{0}, r_{1}, \ldots, r_{m-1}\right)$ actually represents the value $\left(r_{0}, r_{1}, \ldots, r_{m-1-s(\lceil(t+2) / s\rceil-1)}\right)$ regardless of the value of the last $s(\lceil(t+2) / s\rceil-1)$ elements. Therefore, we get

$$
V=\left(\frac{t !}{(t-s) !}\right)^{\frac{m}{s}-\left\lceil\frac{t+2}{s}\right\rceil+1}
$$

We also denote $L=\operatorname{lcm}\left(m, V^{m-1}\right)$.

Consider a de-Bruijn sequence $S$ of order $m-1$ over the alphabet $\{0,1, \ldots, V-1\}$. The Gray code $\bar{G}$ of anchor vectors is a sequence $g_{0}, g_{1}, \ldots, g_{L-1}$ of $L$ mixed-radix vectors of length $m^{2}=n$. Each vector is formed by a concatenation of $m$ blocks of length $m$. Between the anchors $g_{i}$ and $g_{i+1}$, the block $v_{s_{i}}$ is transformed into the block $v_{s_{i+m}}$.

Within each of the $m$ blocks comprising any single anchor, the $(m-2)$ nd digit (the second-from-right digit - a noninformation digit) is set to 1 in all blocks except for the underlined block. For brevity, we call this digit the underline digit.

Between any two anchors, $g_{i}$ and $g_{i+1}$, a sequence of vectors called auxiliary vectors and denoted $g_{i}^{0}, g_{i}^{1}, \ldots, g_{i}^{\ell_{i}}$, is formed by a sequence of push-to-the-top operations on the cells of the changing block. The auxiliary vectors are determined by Algorithm 1 described shortly.

In what follows we present Algorithm 1 that specifies the sequence $g_{i}^{0}, g_{i}^{1}, \ldots, g_{i}^{\ell_{i}}$ that allow us to move from anchor state $g_{i}$ to state $g_{i+1}$. As $g_{i}$ and $g_{i+1}$ differ only in a single block (and this block is changed from $v_{s_{i}}$ to $v_{s_{i+m}}$ ), the same will hold for the sequence $g_{i}^{0}, g_{i}^{1}, \ldots, g_{i}^{\ell_{i}}$, i.e., $g_{i}^{j}$ and $g_{i}^{j^{\prime}}$ will only differ in the block in which $g_{i}$ and $g_{i+1}$ differ. Thus, it suffices to define in Algorithm 1 how to change a block of length $m$ with cell values that represent $v_{s_{i}}$ into a block that represents $v_{S_{i+m}}$ using push-to-the-top operations. However, we call the attention of the reader to the fact that while the change in represented value affects only one block, for administrative reasons we also push cells of the block to the left (cyclically).

We now present Algorithm 1 and describe some of its properties. We then prove that indeed the resulting code $G$ is an asymptotically-optimal cyclic $(s, t, n)$-LRMGC. We assume that the following algorithm is applied to positions $\{0,1, \ldots, m-1\}$. We further assume $\left(r_{0}, r_{1}, \ldots, r_{m}\right) \in([t] \times$ $[t-1] \times \cdots \times[t-s])^{m / s}$ represents the value $v_{\ell}$, then we say the $j$ th digit of $v_{\ell}$ is

$$
v_{\ell}(j)= \begin{cases}r_{j} & 0 \leqslant j<m-s(\lceil(t+2) / s\rceil-1) \\ 0 & \text { otherwise. }\end{cases}
$$

Finally, we restrict $l(\cdot)$ and $r(\cdot)$ by defining

$$
\begin{aligned}
& l^{\prime}(j)= \begin{cases}l(j) & 0 \leqslant l(j) \leqslant m-3 \\
0 & \text { otherwise }\end{cases} \\
& r^{\prime}(j)= \begin{cases}r(j) & 0 \leqslant r(j) \leqslant m-3 \\
m-3 & \text { otherwise }\end{cases}
\end{aligned}
$$

Our algorithm changes a block of length $m$ with cell values that represent $v_{S_{i}}$ into one that represents $v_{S_{i+m}}$ using pushto-the-top operations. It is strongly based on the factoradic representation of $v_{s_{i+m}}$. Let $v_{s_{i+m}}(j)$ be the $j$ th entry in this representation. Namely, if $\mathbf{c}=\left(c_{1}, \ldots, c_{m}\right)$ is a cell configuration that corresponds to $v_{\mathcal{S}_{i+m}}$, then for each index $j \in[m]$ the number of entries in the window corresponding to $j$ that are to the right of $j$ and are of value lower than $c_{j}$ equal $v_{s_{i+m}}(j)$. Roughly speaking, to obtain such a configuration c, our algorithm, for $j \in[m]$, pushes each cell $c_{j}$ in $\mathbf{c}$ to the top 


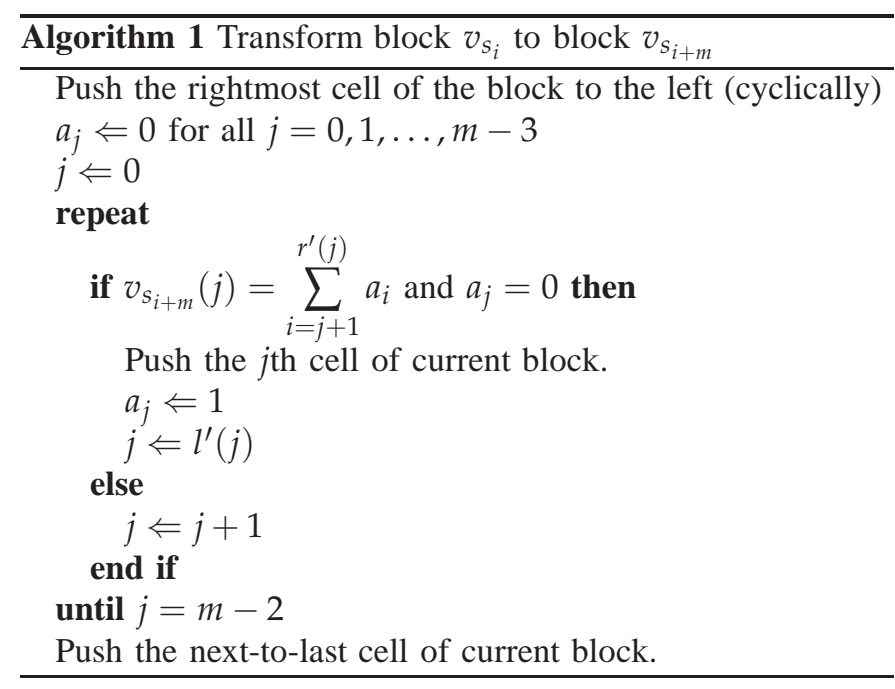

exactly once and only after exactly $j$ cells to the right of $c_{j}$ (and participating in the window corresponding to $j$ ) have been pushed to the top. As each time a cell is changed it is pushed to the top, this will ensure that the resulting cell configuration c will have a factoradic representation corresponding to $v_{S_{i+m}}$.

A few remarks are in place. In order to keep track of which cells were pushed during our algorithm, we save an array of bits $a_{j}$ for each cell in the block (initialized to 0 ), indicating whether the cell $c_{j}$ has been pushed before. We note that in order to be able to decode a state, we need to have some way to know which block is being currently changed, i.e., the imaginary underline in the anchor. We use the last two cells of each block for that purpose.

Example 4. Take the case of $(1,2,16)-L R M$ with $m=4, V=$ 2 , and a de-Bruijn sequence of order 3 and alphabet of size 2 is $S=00010111$. The list of anchors is

$\begin{array}{lllll}g_{0}= & \mathbf{1 0 1 0} & \mathbf{0 0 1 0} & \mathbf{0 0 1 0} & \underline{\mathbf{0 0 0 0}} \\ g_{1}= & \mathbf{1 0 1 0} & \mathbf{0 0 1 0} & \underline{\mathbf{0 0 0 0}} & \mathbf{0 0 1 0} \\ g_{2}= & \mathbf{1 0 1 0} & \underline{\mathbf{0 0 0 0}} & \underline{\mathbf{1 0 1 0}} & \mathbf{0 0 1 0} \\ g_{3}= & \underline{\mathbf{1 0 0 0}} & \underline{\mathbf{1 0 1 0}} & \mathbf{1 0 1 0} & \mathbf{0 0 1 0} \\ g_{4}= & \mathbf{1 0 1 0} & \mathbf{1 0 1 0} & \mathbf{1 0 1 0} & \underline{\mathbf{0 0 0 0}} \\ g_{5}= & \mathbf{1 0 1 0} & \mathbf{1 0 1 0} & \underline{\mathbf{1 0 0 0}} & \mathbf{0 0 1 0} \\ g_{6}= & \mathbf{1 0 1 0} & \underline{\mathbf{1 0 0 0}} & \mathbf{0 0 1 0} & \mathbf{0 0 1 0} \\ g_{7}=\underline{\mathbf{1 0 0 0}} & \mathbf{0 0 1 0} & \mathbf{0 0 1 0} & \mathbf{0 0 1 0}\end{array}$

The bold bit (the leftmost bit in each group of four) denotes the information bit, while the rest are non-information bits. We note that the underlined vectors are easily recognizable by nextto-right bit being 0 .

Notice that in this example the information bit is dominated in size by the remaining bits of each block. This is an artifact of our example in which we take $n$ be be small. For large values of $n$ the overhead in each block is negligible with respect to the information bits.

As an example, the transition between $g_{1}$ and $g_{2}$ is (the changed positions are underlined)

$\begin{array}{lllll}g_{1}= & 1010 & 0010 & 0000 & 0010 \\ g_{1}^{0}= & 1010 & 0001 & 0000 & 0010 \\ g_{1}^{1}= & 1010 & 0001 & 0 \underline{100} & 0010 \\ g_{1}^{2}= & 1010 & 000 \underline{0} & \underline{1100} & 0010 \\ g_{2}= & 1010 & 0000 & \underline{1010} & 0010\end{array}$

We now address the analysis of Algorithm 1

Lemma 5. Assuming the underline is known, all anchors used in Construction 1 are distinct.

Proof: Proof follows directly from the properties of the de-Bruijn sequence $S$ and the fact that we are taking $L$ to be the $\operatorname{lcm}\left(m, V^{m-1}\right)$.

Lemma 6. Algorithm 1 maintains the correctness of the underline digit in anchors. In addition, between any two adjacent anchors, Algorithm 1 guarantees the underline digits of the changing block and the block to its left (cyclically), are both not maximal.

Proof: Proof is by induction. The base case follows from our construction of the first anchor element $g_{0}$. Assume $g_{i}$ satisfies the inductive claim. When applying Algorithm 1 to move from anchor $g_{i}$ to $g_{i+1}$, we start by pushing the rightmost cell of the block to the left of that being changed. This implies that the value of the underline cell in both the block being changed and that to its left are now not maximal. This state of affairs remains until the end of Algorithm 1, in which we push the next-to-the last cell in the changed block. At that point in time, the underline cell in the changed block obtains it's maximal value, while the block to its left (that to be changed in the next application of Algorithm 10 is of non-maximal value. All the other underline cells remain unchanged throughout the execution of Algorithm 1

Lemma 7. Algorithm 1 terminates, and when it does, all of the cells are pushed exactly once.

Proof: That Algorithm 1 terminates is easy to see. For convenience, we denote $z=s(\lceil(t+2) / s\rceil-1)$. For each $k \in\{m-z, \ldots, m-3\}, v_{s_{i+m}}(k)=0$, and therefore each of those cells is pushed the first time that $j=k$. Now we assume by induction that for each $k \leqslant m-z$, all of the cells with indices $j, k \leqslant j \leqslant m-3$, are pushed before the algorithm terminates.

The base case, $k=m-z$, was already proved above. For the induction step, by the induction assumption, we know that all the cells in $\left\{k, \ldots, r^{\prime}(k)\right\}$ are pushed. At the point where exactly $v_{s_{i+m}}(k-1)$ of them are pushed, cell $k-1$ is pushed in the next visit. Since also the algorithm never pushes a cell more than once, the claim is proved.

Theorem 8. Algorithm 1 changes a block representing $v_{s_{i}}$ into a block representing $v_{S_{i+m}}$.

Proof: Before cell $j$ is being pushed, exactly $v_{s_{i+m}}(j)$ cells from $\left\{j+1, \ldots, r^{\prime}(j)\right\}$ have been pushed already. The 
rest will be pushed after and above it, and therefore its rank is exactly $v_{S_{i+m}}(j)$, as desired.

One drawback of Algorithm 1 is that it may visit a codeword multiple times. For example, assume a $(1,2,25)$-LRM scheme, with $v_{s_{i}}=11 X X X$ and $v_{s_{i+5}}=10 X X X$, where $X$ is the "don't care" symbol. The algorithm would, after an initial push of a cell on the adjacent block to the left, first push cell 1, changing the block state to $01 X X X$. Afterwards, the algorithm would push cell 0 , changing the state back to $v_{s_{i}}$.

To solve that problem, we suggest to simulate the entire remaining execution of the algorithm every time we push a cell. If the resulting configuration after the planned push appears another time in the future, we change the algorithm's inner state to that of the latest such repeat appearance. That way we make sure that each codeword appears only once in the Gray code. We call the revised algorithm the repetitionavoiding algorithm.

Lemma 9. The time complexity of the repetition-avoiding algorithm is $O(t n)$.

Proof: Each cell is visited by the algorithm at most $t$ times, once during the first visit of the algorithm, and once following each of the $t-1$ cells immediately to its right being pushed. Since each cell is pushed exactly once, a full execution of the algorithm takes $\mathrm{O}(\mathrm{tm})$ steps. For the repetition-avoiding algorithm, simulating a full execution after each push results in total time complexity $O\left(t m+t m^{2}\right)=O(t n)$.

Combining all of our observations up to now, we are able to summarize with the following theorem for $G$ from Construction 1

Theorem 10. $G$ is a cyclic gray code of size at least $L$.

Corollary 11. For all constants $1 \leqslant s<t$, there exists an asymptotically-optimal family of codes, $\left\{G_{i}\right\}_{i=t+2}^{\infty}$, where $G_{i}$ is an $\left(s, t, n_{i}\right)$-LRMGC of size $N_{i}, n_{i+1}>n_{i}$, with

$$
\lim _{i \rightarrow \infty} \frac{\log _{2} N_{i}}{\log _{2}\left|\mathcal{R}\left(s, t, n_{i}\right)\right|}=1 \text {. }
$$

Proof: We set $n_{i}=s^{2} i^{2}$ for all $i \geqslant t+2$. Then $N_{i} \geqslant$ $L_{i} \geqslant V_{i}^{s i-1}$. It follows that

$$
\begin{aligned}
& \lim _{i \rightarrow \infty} \frac{\log _{2} N_{i}}{\log _{2}\left|\mathcal{R}\left(s, t, n_{i}\right)\right|} \geqslant \\
& \geqslant \lim _{i \rightarrow \infty} \frac{(s i-1) \log _{2} V_{i}}{\log _{2}\left((t-s) ! \cdot\left(\frac{t !}{(t-s) !}\right)^{s i^{2}}\right)} \\
& =\lim _{i \rightarrow \infty} \frac{(s i-1) \log _{2}\left(\frac{t !}{(t-s) !}\right)^{i-\left\lceil\frac{t+2}{s}\right\rceil+1}}{\log _{2}\left((t-s) ! \cdot\left(\frac{t !}{(t-s) !}\right)^{s i^{2}}\right)} \\
& =1 .
\end{aligned}
$$

\section{CONCLusions}

We presented the framework for $(s, t, n)$-local rank modulation, and studied Gray codes for the most general case. The codes we present are asymptotically optimal.

Several questions remain open. For the case of $(1,2, n)$ LRM, a previous work describes asymptotically-optimal codes for which the weight of the codewords is constant and approaches $\frac{n}{2}$ [9]. That property guarantees a bounded charge difference in any "push-to-the-top" operation. Constant-weight codes for the general case are still missing. Of more general interest is the study of codes that cover a constant fraction of the space.

\section{REFERENCES}

[1] T. Berger, F. Jelinek, and J. K. Wolf, "Permutation codes for sources," IEEE Trans. on Inform. Theory, vol. IT-18, no. 1, pp. 160-169, Jan. 1972.

[2] I. F. Blake, "Permutation codes for discrete channels," IEEE Trans. on Inform. Theory, vol. 20, pp. 138-140, 1974.

[3] I. F. Blake, G. Cohen, and M. Deza, "Coding with permutations," Inform. and Control, vol. 43, pp. 1-19, 1979.

[4] V. Bohossian, A. Jiang, and J. Bruck, "Buffer coding for asymmetric multi-level memory," in Proceedings of the 2007 IEEE International Symposium on Information Theory (ISIT2007), Nice, France, Jun. 2007, pp. 1186-1190.

[5] H. Chadwick and I. Reed, "The equivalence of rank permutation codes to a new class of binary codes," IEEE Trans. on Inform. Theory, vol. 16, no. 5, pp. 640-641, 1970.

[6] H. D. Chadwick and L. Kurz, "Rank permutation group codes based on Kendall's correlation statistic," IEEE Trans. on Inform. Theory, vol. IT-15, no. 2, pp. 306-315, Mar. 1969.

[7] G. Cohen and M. Deza, "Decoding of permutation codes," in Intl. CNRS Colloquium, July, France, 1977.

[8] M. Deza and P. Frankl, "On maximal numbers of permutations with given maximal or minimal distance," J. Combin. Theory Ser. A, vol. 22, 1977.

[9] E. En Gad, M. Langberg, M. Schwartz, and J. Bruck, "On a construction for constant-weight gray codes for local rank modulation," in Proceedings of the 2010 IEEE 26-th Convention of Electrical and Electronic Engineers in Israel (IEEEI2010), Eilat, Israel, Nov. 2010, p. 996.

[10] H. C. Ferreira, A. J. H. Vinck, T. G. Swart, and I. de Beer, "Permutation trellis codes," IEEE Trans. on Communications, pp. 1782-1789, Nov. 2005.

[11] S. W. Golomb, Shift Register Sequences. Holden-Day, San Francisco, 1967.

[12] A. Jiang, V. Bohossian, and J. Bruck, "Floating codes for joint information storage in write asymmetric memories," in Proceedings of the 2007 IEEE International Symposium on Information Theory (ISIT2007), Nice, France, Jun. 2007, pp. 1166-1170.

[13] A. Jiang and J. Bruck, "Joint coding for flash memory storage," in Proceedings of the 2008 IEEE International Symposium on Information Theory (ISIT2008), Toronto, Canada, Jul. 2008, pp. 1741-1745.

[14] A. Jiang, M. Langberg, M. Schwartz, and J. Bruck, "Universal rewriting in constrained memories," in Proceedings of the 2009 IEEE International Symposium on Information Theory (ISIT2009), Seoul, Korea, Jun. 2009, pp. 1219-1223.

[15] A. Jiang, R. Mateescu, M. Schwartz, and J. Bruck, "Rank modulation for flash memories," IEEE Trans. on Inform. Theory, vol. 55, no. 6, pp. 2659-2673, Jun. 2009.

[16] A. Jiang, M. Schwartz, and J. Bruck, "Correcting charge-constrained errors in the rank-modulation scheme," IEEE Trans. on Inform. Theory, vol. 56, no. 5, pp. 2112-2120, May 2010.

[17] C. A. Laisant, "Sur la numération factorielle, application aux permutations," Bulletin de la Société Mathématique de France, vol. 16, pp. 176-183, 1888.

[18] M. Schwartz, "Constant-weight Gray codes for local rank modulation," in Proceedings of the 2010 IEEE International Symposium on Information Theory (ISIT2010), Austin, TX, U.S.A., Jun. 2010, pp. 869-873. 
[19] D. Slepian, "Permutation modulation," in Proc. of the IEEE, vol. 53, no. 3,1965 , pp. $228-236$.

[20] I. Tamo and M. Schwartz, "Correcting limited-magnitude errors in the rank-modulation scheme," IEEE Trans. on Inform. Theory, vol. 56, no. 6, pp. 2551-2560, Jun. 2010.

[21] H. Vinck, J. Haering, and T. Wadayama, "Coded M-FSK for power line communications," in Proceedings of the 2000 IEEE International Symposium on Information Theory (ISIT2000), Sorrento, Italy, 2000, p. 137.

[22] Z. Wang, A. Jiang, and J. Bruck, "On the capacity of bounded rank modulation for flash memories," in Proceedings of the 2009 IEEE International Symposium on Information Theory (ISIT2009), Seoul, Korea, Jun. 2009, pp. 1234-1238.

[23] E. Yaakobi, P. H. Siegel, and J. K. Wolf, "Buffer codes for multilevel flash memory," in Proceedings of the 2008 IEEE International Symposium on Information Theory (ISIT2008), Toronto, Canada, 2008, poster. 